\title{
Risk for Death among Children with Pneumonia, Afghanistan
}

\author{
Rahmani Zabihullah, ${ }^{1}$ Bhim G. Dhoubhadel, ${ }^{1}$ \\ Ferogh A. Rauf, Sahab A. Shafiq, Motoi Suzuki, \\ Kiwao Watanabe, Lay M. Yoshida, Michio \\ Yasunami, Salihi Zabihullah, Christopher M. \\ Parry, Rabi Mirwais, Koya Ariyoshi
}

In Afghanistan, childhood deaths from pneumonia are high. Among 639 children at 1 hospital, the case-fatality rate was $12.1 \%$, and $46.8 \%$ of pneumococcal serotypes detected were covered by the 13-valent vaccine. Most deaths occurred within 2 days of hospitalization; newborns and malnourished children were at risk. Vaccination could reduce pneumonia and deaths.

$I^{\prime}$ n Afghanistan, the mortality ratio for children $<5$ years Lof age is 90 deaths $/ 1,000$ live births, twice the global average; $20 \%$ of deaths are from pneumonia (1). Although Afghanistan is considered 1 of the 5 countries with the highest level of childhood deaths from pneumonia, studies of the risk factors for death and etiology of pneumonia among children in Afghanistan are lacking (2). We therefore determined risk factors for death from pneumonia in children $<5$ years of age in a regional hospital in Afghanistan and the distribution of pneumococcal serotypes carried in the nasopharynx.

\section{The Study}

From December 2012 through the second week of March 2013, we conducted a prospective observational study in the Department of Pediatrics in Abu Ali Sina Balkhi Regional Hospital, Mazar-e-Sharif, Afghanistan, a 700-bed regional referral hospital for Balkh Province. This study was conducted before pneumococcal conjugate vaccine 13 (PCV13) had been introduced in Afghanistan. We enrolled children $<5$ years of age who met the World Health Organization (WHO) criteria for clinical pneumonia at the time of admission (3). We collected data by standardized

Author affiliations: Nagasaki University, Nagasaki, Japan (R. Zabihullah, B.G. Dhoubhadel, M. Suzuki, K. Watanabe, L.M. Yoshida, M. Yasunami, C.M. Parry, K. Ariyoshi); Abu Ali Sina Balkhi Regional Hospital, Mazar-e-Sharif, Afghanistan (F.A. Rauf, S.A. Shafiq, S. Zabihullah); London School of Hygiene and Tropical Medicine, London, UK (C.M. Parry); Public Health Department, Balkh Province, Afghanistan (R. Mirwais) questionnaire and determined immunization status by history, immunization report, and bacillus Calmette-Guérin (BCG) scar.

Malnutrition was defined as weight-for-age $z$ score $<-2$ (WHO Anthro software version 3.2.2, http://www.who.int/ childgrowth/software/en/). Anemia in children $>6$ months of age was determined by hemoglobin cutoff values established by WHO (4) and in children $\leq 6$ months by hemoglobin value $<2$ SDs below the mean for age group (5). Illness severity was classified by WHO criteria (3). Hospital outcomes were classified as discharged (discharged after successful treatment), deceased (died during hospitalization), and unknown (still in hospital at study end). Children with unknown status were excluded from risk factor analysis. Neonates/newborns and infants were defined as children $<1$ and $<12$ months of age, respectively.

We collected nasopharyngeal samples according to the WHO protocol by using flocked swabs (Copan Diagnostics, Murireta, CA, USA) that were stored in STGG (skim milk, tryptone, glucose, and glycerine) media at $-10^{\circ} \mathrm{C}$ and transferred within 1 month by airplane with cold ice packs in a thermos to Nagasaki, Japan, where they were stored at $-20^{\circ} \mathrm{C}$. DNA was extracted by using a QIAamp DNA Blood Mini Kit (QIAGEN, Hilden, Germany). Streptococcus pneumoniae was detected by lytA real-time PCR (selective for autolysin gene), and serotyping was performed by a nanofluidic real-time PCR that detects 50 serotypes as individual serotype/serogroup including all vaccine serotypes (6).

We considered samples positive by lytA PCR but negative for serotypes/serogroups nontypeable and cocolonization with multiple serotypes to be presence of $\geq 2$ serotypes/serogroups in a sample. The percentage of vaccine serotypes was calculated as the proportion of samples that had a vaccine serotype (including minor serotypes in co-colonization) among samples positive by lytA PCR.

For analyses we used Epi Info version 7 (Centers for Disease Control and Prevention, Atlanta, GA, USA) and Stata 12 (StataCorp LLP, College Station, TX, USA). In the univariate analysis model, we included risk factors for death from pneumonia at $\mathrm{p}<0.2$, and in the multivariate model we included age, sex, and ethnicity. The study complied with STROBE (https://strobe-statement.org/ fileadmin/Strobe/uploads/checklists/STROBE_checklist_

\footnotetext{
${ }^{1}$ These authors contributed equally to this article.
} 
v4_combined.pdf) guidelines and was approved by the Nagasaki University Institutional Review Board, Nagasaki, Japan, and the Balkh Public Health Department, Balkh, Afghanistan. Written informed consent was obtained from the parents of enrolled children.

Parents of 670 children were approached, and 639 children were enrolled (online Technical Appendix Figure 1, https://wwwnc.cdc.gov/EID/article/23/8/15-1550Techapp1.pdf). Median patient age was 5.0 (interquartile range 2.5-9.0) months; $82.5 \%$ were infants and $64.3 \%$ were male (Table 1). Pneumonia case-fatality ratio (CFR) was $12.1 \%(75 / 617$; 95\% CI 9.6\%-14.9\%) (online Technical Appendix Table 1). A total of $61(81.3 \%)$ children

\begin{tabular}{|c|c|}
\hline Characteristics & No. $(\%)$ \\
\hline \multicolumn{2}{|l|}{ Sex } \\
\hline M & $411(64.3)$ \\
\hline $\mathrm{F}$ & $228(35.7)$ \\
\hline \multicolumn{2}{|l|}{ Age, mo } \\
\hline$<1$ (newborn) & $17(2.7)$ \\
\hline $1-11$ & $510(79.8)$ \\
\hline$\geq 12$ & $112(17.5)$ \\
\hline Māernal illiteracy & $549(85.9)$ \\
\hline Duration of illness $>7 \mathrm{~d}$ before hospitalization & $102(16.0)$ \\
\hline \multicolumn{2}{|l|}{ Ethnicity } \\
\hline Tajik & $300(46.9)$ \\
\hline Pashtoon & $123(19.3)$ \\
\hline Uzbek & $77(12.0)$ \\
\hline Hazara & $101(15.8)$ \\
\hline Other & $38(6.0)$ \\
\hline \multicolumn{2}{|l|}{ Received antimicrobial drugs before hospitalization } \\
\hline Yes & $561(87.8)$ \\
\hline No & $65(10.2)$ \\
\hline Unknown & $13(2.0)$ \\
\hline \multicolumn{2}{|c|}{ Vaccination status, vitamin A intake, and nutritional status } \\
\hline Bacillus Calmette-Guérin vaccine & $545(85.2)$ \\
\hline$\geq 1$ dose of pentavalent vaccine, $n=568$ & $436(76.8)$ \\
\hline Measles vaccine, $n=171$ & $107(62.6)$ \\
\hline$\geq 1$ dose of vitamin $A, n=345$ & $119(34.5)$ \\
\hline \multicolumn{2}{|l|}{ Malnutrition* } \\
\hline Detected & $255(39.9)$ \\
\hline Not detected & $381(59.6)$ \\
\hline Not evaluated & $3(0.5)$ \\
\hline \multicolumn{2}{|l|}{ Anemia† } \\
\hline Detected & $296(46.3)$ \\
\hline Not detected & $220(34.4)$ \\
\hline Not evaluated & $123(19.3)$ \\
\hline Both malnutrition and anemia, $\mathrm{n}=514$ & $126(24.5)$ \\
\hline Very severe pneumonia & $532(83.3)$ \\
\hline \multicolumn{2}{|l|}{ Clinical outcome, $n=617$} \\
\hline Discharged well & $542(87.9)$ \\
\hline Death & $75(12.1)$ \\
\hline
\end{tabular}

*Defined by weight/age $z$ score $<-2$, and the $z$ score value was calculated by using World Health Organization Anthro software, version 3.2.2 9 (http://www.who.int/childgrowth/software/en/).

†Defined according to the World Health Organization (4) and Janus et al. (5).

¥Severity classified according to World Health Organization (3). Very severe pneumonia is defined as cough or difficulty breathing with 1 of the following: central cyanosis, difficulty feeding, convulsions, lethargy, loss of consciousness, severe respiratory distress.

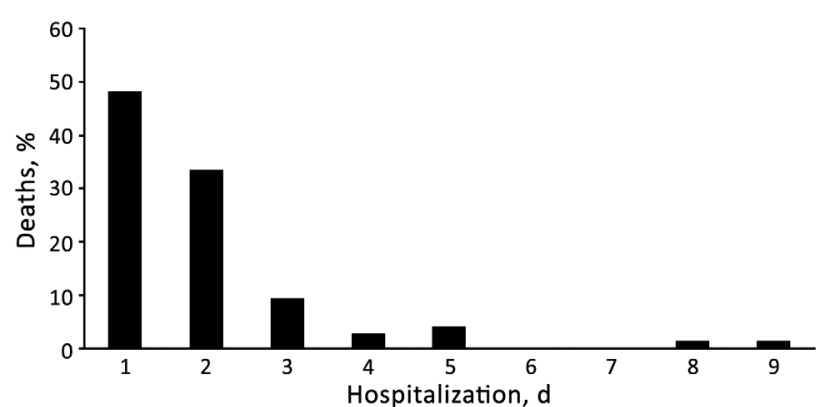

Figure 1. Proportion of deaths and days of hospitalization among children $<5$ years of age with pneumonia admitted to Abu Ali Sina Balkhi Regional Hospital, Mazar-e-Sharif, Afghanistan, December 2012-March 2013.

died within 2 days of hospitalization (Figure 1), and most were infants (online Technical Appendix Figure 2).

According to univariate analysis, risk for death was increased among newborn (odds ratio [OR] 11.1) and malnourished (OR 2.06) children (Table 2). Protective factors were receipt of BCG vaccine (OR 0.39), $\geq 1$ dose of pentavalent vaccine (OR 0.53), and vitamin A (OR 0.39). Among malnourished children, female sex was associated with death (online Technical Appendix Table 2). We found no significant differences by sex in terms of epidemiologic, clinical, and nutritional status (data not shown). BCG vaccination was independently associated with decreased risk for death among patients with pneumonia (Table 2) and among malnourished children with pneumonia (online Technical Appendix Table 2).

We obtained nasopharyngeal samples from 326 children (online Technical Appendix Figure 1). From half (49.9\%) of the children, samples could not be taken because of disease severity; CFR was $18.3 \%$ for these children, who were more likely to be malnourished and to have received antimicrobial drugs before hospitalization (online Technical Appendix Table 3). S. pneumoniae was detected in 124 $(38.0 \%)$ of the 326 samples; 24 serotypes/serogroups were identified (Figure 2). Most (87.8\%) children had received antimicrobial drugs before admission, which, along with difficulty storing samples at the research site, influenced detection of pneumococci. The proportions of colonization were $35.9 \%$ among children who received antimicrobial drugs before hospitalization and $48.8 \%$ among children who did not $(\mathrm{p}=0.11)$. The proportion of samples that had serotypes covered by 7-, 10-, and 13-valent pneumococcal conjugate vaccines were $39.5 \%, 39.5 \%$ and $46.8 \%$, respectively. Co-colonization with multiple serotypes occurred in $21(16.9 \%)$ of 124 positive samples.

\section{Conclusions}

The CFR for children $<5$ years of age with pneumonia admitted to a regional hospital in Afghanistan was 12.1\%, 


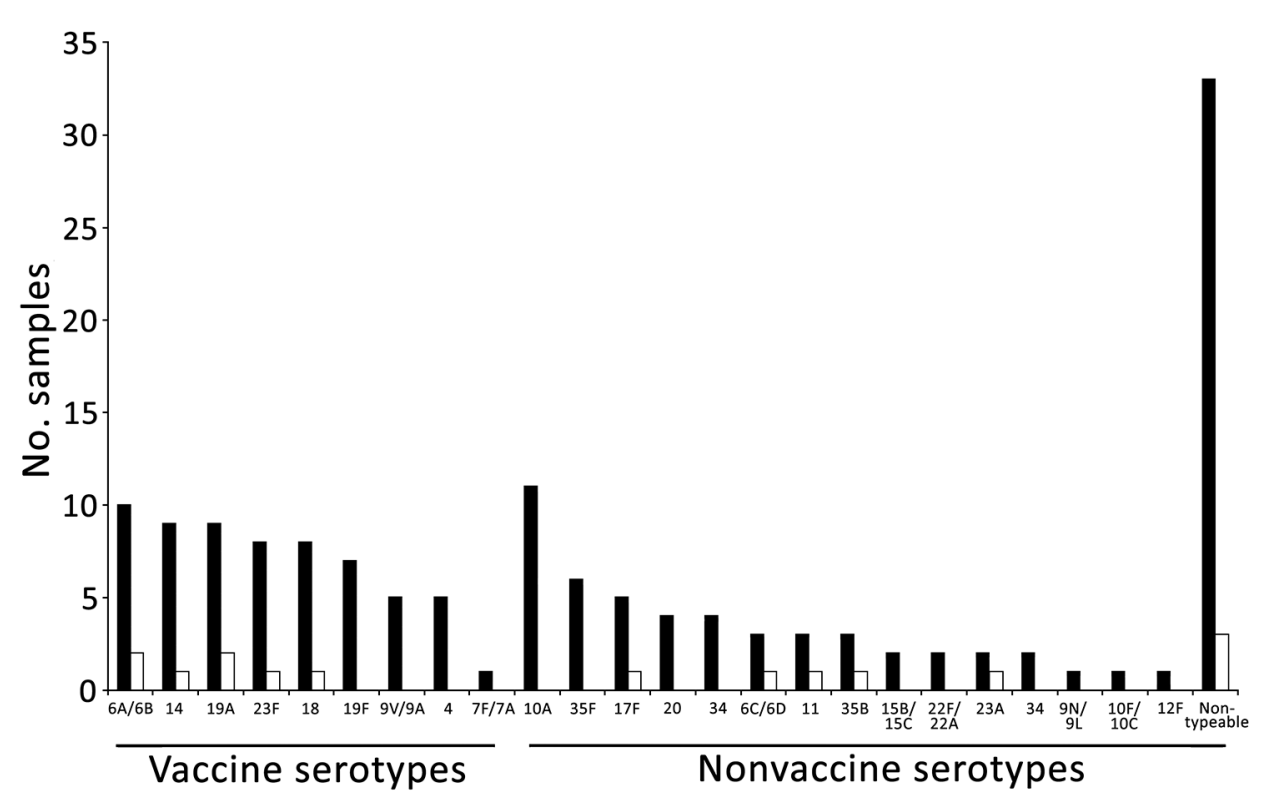

Figure 2. Number of nasopharyngeal samples and pneumococcal serotype/ serogroup distribution (including minor serotypes in multiple serotypes) among 110 discharged (black bars) and 11 deceased (white bars) children with pneumonia admitted to Abu Ali Sina Balkhi Regional Hospital, Mazar-e-Sharif, Afghanistan, December 2012March 2013. compared with only $7.6 \%$ for the full WHO Eastern Mediterranean region (7). Most deaths occurred within 2 days of hospitalization. Factors that may have contributed to the high mortality rate were delays in presentation to healthcare facilities, inability to identify severe symptoms in children, and delayed referral from primary care (7). These issues could be addressed by strengthening the Integrated Management of Childhood Illness program of WHO, introduced in Afghanistan in 2004 (8).

Our finding that newborns and children with malnutrition were at increased risk for death is consistent with findings of studies in India and Pakistan $(9,10)$. These risks could be reduced by use of clean delivery kits, clean delivery practices, exclusively breast-feeding, education about complementary feeding, and provision of complementary foods in regions where food is less secure $(11,12)$.

BCG vaccination was protective. Neonatal BCG vaccination is known to be associated with reduced rates of childhood death, respiratory infection, and sepsis, probably by nonspecific immune effects (13); it can also be a proxy for better access to healthcare, immunization, and unmeasured favorable factors.

Female sex was significantly associated with death among malnourished children, which was not explained by association with other variables. A higher incidence of acute lower respiratory infection in male children, particularly in southern Asia, was reported in a recent systematic review, but the pneumonia CFR was higher in girls than in boys $<1$ year of age (7).

With support from the Global Vaccine Alliance, WHO, and the United Nations Children's Fund, PCV13 was introduced in Afghanistan in January 2014. Our study detected a wide variety of serotypes, including nonvaccine serotypes. Vaccine coverage was comparable with that found by regional studies $(14,15)$. Serotype data are limited by the short study duration, common use of antimicrobial drugs before hospitalization, and difficult storage of samples.

The high rate of death from pneumonia among children could be reduced by strengthening existing public health programs (e.g., Integrated Management of Childhood Illness, nutrition programs, and immunization programs). Although the proportion of serotypes covered by PCV13 vaccines was $46.8 \%$, PCV13 could still prevent many cases of pneumonia and deaths among children in Afghanistan.

\section{Acknowledgments}

We are grateful to the children and their parents who participated in this study. We thank the staff of Abu Ali Sina Balkhi Regional Hospital who helped conduct this research.

The study was funded by the Japan Ministry of Education, Culture, Sports, Science, and Technology. R.Z. and B.G.D. are grateful to the government of Japan, Ministry of Education, Culture, Sports, Science, and Technology for their scholarships.

Dr. Rahmani Zabihullah is a medical doctor from Afghanistan; he performed this work for his Master of Tropical Medicine thesis at Nagasaki University. He works as a health coordinator in Japan International Cooperation Agency, Afghanistan, focusing on improvement of children's health.

\section{References}

1. World Health Organization. Afghanistan: health profile. 2014 [cited 2014 May 24]. http://www.who.int/gho/countries/afg.pdf 
Table 2. Risk factors for death from pneumonia among children $<5$ years of age hospitalized at Abu Ali Sina Balkhi Regional Hospital, Mazar-e-Sharif, Afghanistan, December 2012-March 2013

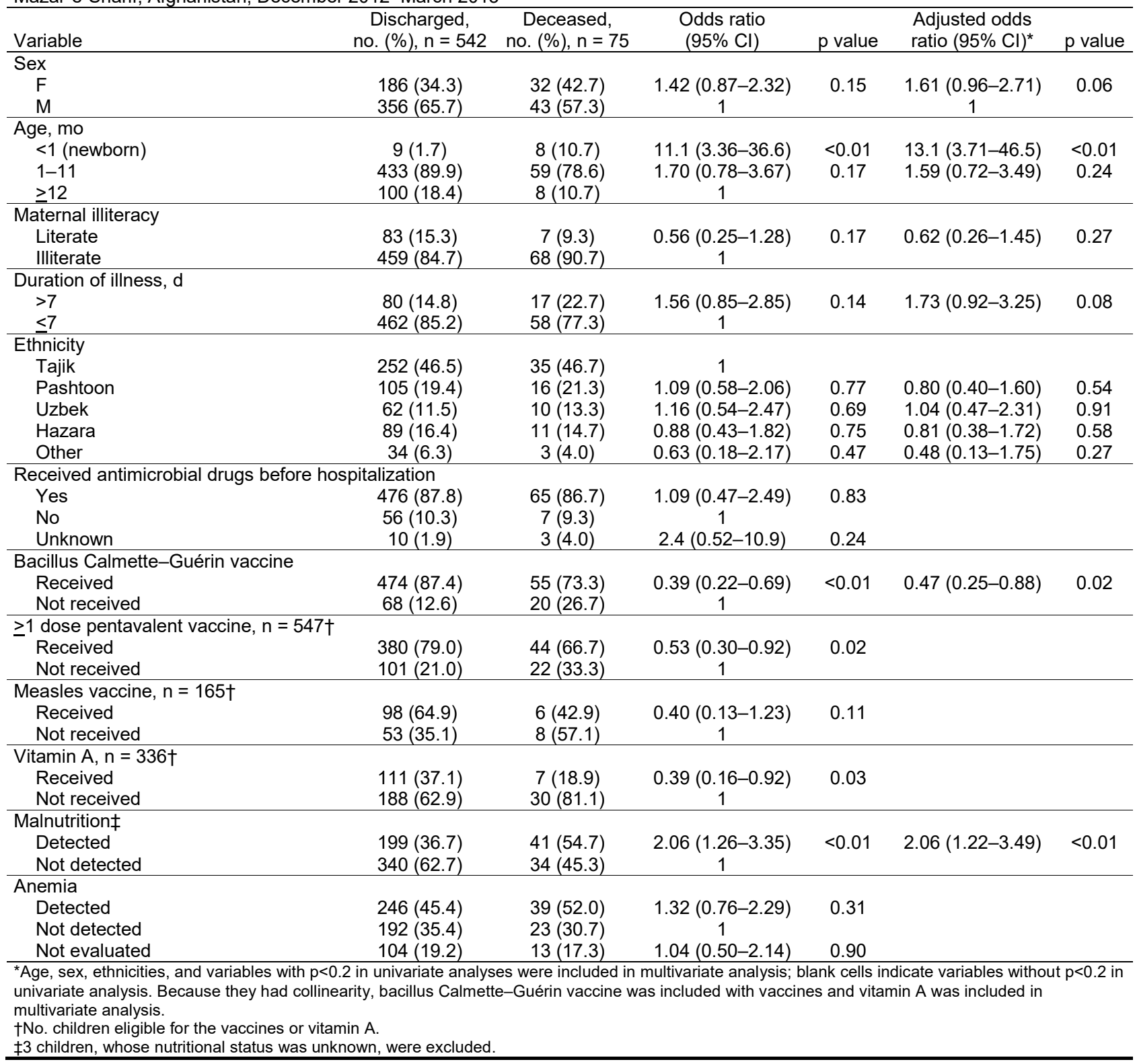

2. Gilani Z, Kwong YD, Levine OS, Deloria-Knoll M, Scott JA, O'Brien KL, et al. A literature review and survey of childhood pneumonia etiology studies: 2000-2010. Clin Infect Dis. 2012;54(Suppl 2):S102-8. http://dx.doi.org/10.1093/cid/cir1053

3. World Health Organization. Pocket book of hospital care for children: guidelines for the management of common illnesses with limited resources. Geneva: The Organization; 2005.

4. World Health Organization. Haemoglobin concentrations for the diagnosis of anaemia and assessment of severity. Vitamin and mineral nutrition information system. WHO/NMH/NHD/ MNM/11.1. Geneva: The Organization; 2011.

5. Janus J, Moerschel SK. Evaluation of anemia in children. Am Fam Physician. 2010;81:1462-71.

6. Dhoubhadel BG, Yasunami M, Yoshida LM, Thi HA, Thi TH, Thi TA, et al. A novel high-throughput method for molecular serotyping and serotype-specific quantification of Streptococcus pneumoniae using a nanofluidic real-time PCR system. J Med Microbiol. 2014;63:528-39. http://dx.doi.org/10.1099/ jmm.0.071464-0

7. Nair H, Simões EA, Rudan I, Gessner BD, Azziz-Baumgartner E, Zhang JS, et al.; Severe Acute Lower Respiratory Infections Working Group. Global and regional burden of hospital admissions for severe acute lower respiratory infections in young children in 2010: a systematic analysis. Lancet. 2013; 381:1380-90. http://dx.doi.org/10.1016/S0140-6736(12)61901-1

8. Bryce J, Victora CG, Habicht JP, Black RE, Scherpbier RW; MCE-IMCI Technical Advisors. Programmatic pathways to child survival: results of a multi-country evaluation of Integrated Management of Childhood Illness. Health Policy Plan. 2005;20(Suppl 1):i5-17. http://dx.doi.org/10.1093/heapol/czi055 
9. The Million Death Study Collaborators. Causes of neonatal and child mortality in India: a nationally representative mortality survey. Lancet. 2010;376:1853-60.

10. Mustufa MA, Korejo R, Shahid A, Nasim S. Infection remains a leading cause of neonatal mortality among infants delivered at a tertiary hospital in Karachi, Pakistan. J Infect Dev Ctries. 2014;8:1470-5. http://dx.doi.org/10.3855/jidc.3569

11. Seward N, Osrin D, Li L, Costello A, Pulkki-Brännström AM, Houweling TA, et al. Association between clean delivery kit use, clean delivery practices, and neonatal survival: pooled analysis of data from three sites in South Asia. PLoS Med. 2012;9:e1001180. http://dx.doi.org/10.1371/ journal.pmed.1001180

12. Lassi ZS, Das JK, Zahid G, Imdad A, Bhutta ZA. Impact of education and provision of complementary feeding on growth and morbidity in children less than 2 years of age in developing countries: a systematic review. BMC Public Health. 2013;13(Suppl 3):S13. http://dx.doi.org/10.1186/1471-2458-13-S3-S13
13. de Castro MJ, Pardo-Seco J, Martinón-Torres F. Nonspecific (heterologous) protection of neonatal BCG vaccination against hospitalization due to respiratory infection and sepsis. Clin Infect Dis. 2015;60:1611-9. http://dx.doi.org/10.1093/cid/civ144

14. Factor SH, LaClaire L, Bronsdon M, Suleymanova F, Altynbaeva G, Kadirov BA, et al. Streptococcus pneumoniae and Haemophilus influenzae type B carriage, central Asia. Emerg Infect Dis. 2005;11:1476-9. http://dx.doi.org/10.3201/ eid1109.040798

15. Sanaei Dashti A, Abdinia B, Karimi A. Nasopharyngeal carrier rate of Streptococcus pneumoniae in children: serotype distribution and antimicrobial resistance. Arch Iran Med. 2012;15:500-3.

Address for correspondence: Koya Ariyoshi, Department of Clinical Medicine, Institute of Tropical Medicine, Nagasaki University, Sakamoto 3-7-1 Nagasaki 852-8523, Japan; email: kari@nagasaki-u.ac.jp

\section{June 2016: Respiratory Diseases}

- Debate Regarding Oseltamivir Use for Seasonal and Pandemic Influenza

- Perspectives on West Africa Ebola Virus Disease Outbreak, 2013-2016

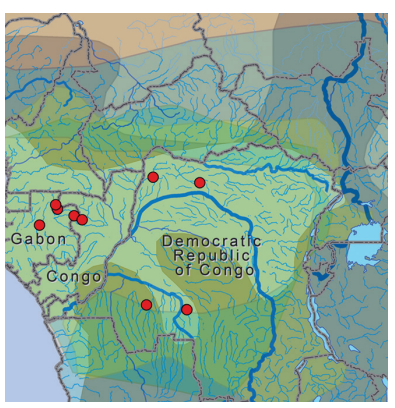

- Human Infection with Influenza A(H7N9) Virus during 3 Major Epidemic Waves, China, 2013-2015

- Integration of Genomic and Other Epidemiologic Data to Investigate and Control a CrossInstitutional Outbreak of Streptococcus pyogenes
- Infectious Disease Risk Associated with Contaminated Propofol Anesthesia, 1989-2014

- Improved Global Capacity for Influenza Surveillance

- Reemergence of Dengue in Southern Texas, 2013

- Transmission of Mycobacterium chimaera from Heater-Cooler Units during Cardiac Surgery despite an Ultraclean Air Ventilation System

- Extended Human-toHuman Transmission during a Monkeypox Outbreak in the Democratic Republic of the Congo

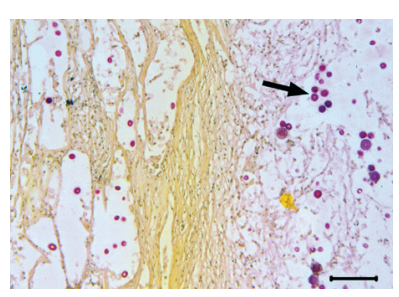

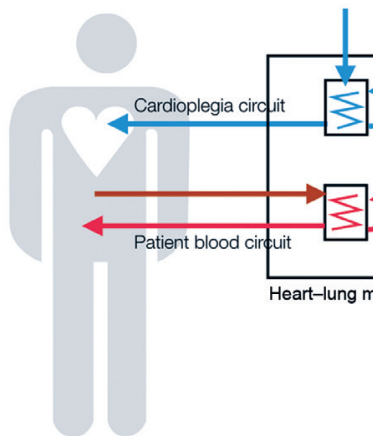

- Use of Population Genetics to Assess the Ecology, Evolution, and Population Structure of Coccidioides, Arizona, USA

- Infection, Replication, and Transmission of Middle East Respiratory Syndrome Coronavirus in Alpacas

- Rapid Detection of Polymyxin Resistance in Enterobacteriaceae

- Human Adenovirus Associated with Severe Respiratory Infection, Oregon,2013-2014
- Heterogeneous and Dynamic Prevalence of Asymptomatic Influenza Virus Infections

- High MICs for Vancomycin and Daptomycin and Complicated CatheterRelated Bloodstream Infections with MethicillinSensitive Staphylococcus aureus

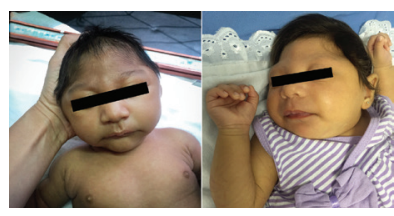

- Population-Level Effect of Cholera Vaccine on Displaced Populations, South Sudan, 2014

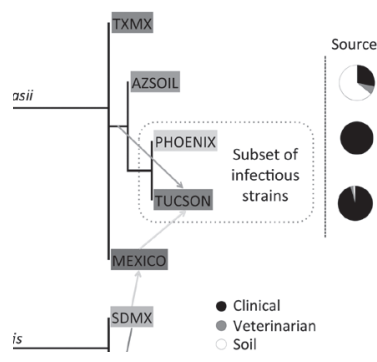

\section{EMERGING INFECTIOUS DISEASES}

https://wwwnc.cdc.gov/eid/articles/ issue/22/6/table-of-contents 